\title{
УДК 517.972.5; 544.45
}

\section{ЧИСЛЕННАЯ ОЦЕНКА КРИТИЧЕСКИХ УСЛОВИЙ В ЗАДАЧЕ О ТЕПЛОВОМ ВЗРЫВЕ В СРЕДЕ С ФЛУКТУАЦИЯМИ РЕАКЦИОННОЙ СПОСОБНОСТИ}

\author{
Донской Игорь Геннадьевич \\ к.т.н., старший научный сотрудник лаборатории термодинамики, \\ e-mail: donskoy.chem@mail.ru, \\ Институт систем энергетики им. Л.А. Мелентьева СО РАН, \\ 664033 г. Иркутск, ул. Лермонтова, 130.
}

\begin{abstract}
Аннотация. Формулируется задача о тепловом взрыве в среде с флуктуациями реакционной способности, которые задаются через функцию распределения энергии активации. Для дифференциального уравнения, описывающего стационарное распределение температуры в такой среде, предложена вариационная формулировка. С использованием простой пробной функции численно исследуется зависимость величины вариационного функционала от максимальной температуры. Критические условия теплового взрыва соответствуют появлению точки перегиба на этой зависимости. Расчеты показывают, что увеличение дисперсии распределения реакционной способности снижает устойчивость системы за счет уменьшения эффективной энергии активации для процесса тепловыделения.
\end{abstract}

Ключевые слова: тепловой взрыв, обратная вариационная задача, критические условия, численное решение.

Цитирование: Донской И. Г. Численная оценка критических условий в задаче о тепловом взрыве в среде с флуктуациями реакционной способности //Информационные и математические технологии в науке и управлении. 2021. № 1 (21). C. 54-65. DOI:10.38028/ESI.2021.21.1.005

Введение. Тепловой взрыв происходит при нарушении баланса между теплоотводом и производством теплоты в активных средах, в первую очередь, в химических реакторах [1]. Прогнозирование условий теплового взрыва составляет важную задачу при проектировании аппаратов химической технологии и управления ими [2]. В данной работе рассматривается одномерная стационарная задача о распределении температуры в реакционноспособной среде со специальными свойствами. Для определения критических условий, как будет показано ниже, можно сформулировать задачу на существование экстремума интегрального функционала.

1. Обзор литературы. Вариационные методы разработаны для исследования процессов с диссипацией: электропроводностью, теплопроводностью, диффузией и вязким течением [4-6]. Даже в этих случаях, однако, возникают проблемы, связанные с полнотой описания и условиями стационарности [7, 8]. Для процессов вблизи равновесия существование вариационных принципов связано с выполнением соотношений линейной неравновесной термодинамики, однако в некоторых случаях эти связи могут быть распространены на нелинейную область $[9,10]$. Известно, что условия химического равновесия определяются экстремумом термодинамического потенциала, вид которого зависит от условий обмена энергией и массой между физико-химической системой и окружением [11]. Аналитическое исследование задач химического равновесия возможно лишь в простейших случаях, однако для практических расчетов разработаны эффективные вычислительные методы (как правило, это методы решения задач нелинейной оптимизации).

Намного сложнее дело обстоит с применением вариационных методов в химической кинетике. Не существует общего метода построения потенциальной функции, градиент которой давал бы уравнения для скоростей химических реакций. В некоторых работах предлагаются различные варианты (часто довольно абстрактные) для построения такого «кинетического потенциала» $[12$ - 14]. Часто эти потенциалы пытаются связать с 
термодинамическими свойствами системы (по аналогии со связью динамических и статических свойств механических систем) [15 - 17]. Авторами работ $[18,19]$ предложен оригинальный метод квазиградиентного описания динамики химических систем, который в явном виде включает параметры равновесного состояния. Другой метод, основанный на использовании новых «термодинамических» переменных при описании каталитических процессов, допускающий экстремальную постановку для стационарного состояния, предложен в [20]. В работах $[21,22]$ предлагается использовать принцип максимального производства энтропии для исследования динамики химических систем, хотя его применимость к этой проблеме не очевидна [23]. В работе [24], напротив, используется принцип минимального производства энтропии, причем результаты доведены до численных расчетов, которые показывают возможность использования этого принципа для редукции кинетических схем.

В работах [25 - 27] предложен вид функционала, минимальность которого выполняется для реализуемой динамики сложных химических систем (что было показано на численных расчетах на основе полной системы кинетических уравнений). Этот функционал имеет связь с термодинамическими характеристиками системы, однако явно зависит от скоростей реакций и производных скоростей реакций от концентраций компонентов. В таком случае вариационный принцип является скорее post hoc-закономерностью: решение экстремальной задачи оказывается сложнее, чем решение прямой кинетической задачи.

Численные методы, основанные на оптимизационных постановках, используются при решении обратных задач $[28,29]$ и для сокращения сложных механизмов химических реакций [30]. Распространение волн реагирования в нелинейных средах можно связать с вариационным принципом, который определяет скорость распространения [31 - 33]. Как указывается в [34], формальная аналогия между стационарными уравнениями реакциидиффузии и уравнениями механики в некоторых случаях позволяет объяснять существование периодических решений в нелинейных системах. Подобным образом в работе [35] предложен вариационный принцип для стационарных систем с простой (линейной) кинетикой.

\section{2. Тепловой взрыв в системе с заданным распределением реакционной} способности. В статье [36], по-видимому, впервые была предложена вариационная формулировка задачи о тепловом взрыве. В приближении Франк-Каменецкого источник теплоты является экспоненциальной функцией температуры, а на стадии предвзрывного разогрева расходованием компонентов можно пренебречь. Квазистационарное распределение температуры удовлетворяет уравнению [1]:

$$
\lambda \frac{d^{2} T}{d x^{2}}+Q \rho k_{0} \exp \left(-\frac{E}{R T}\right)=0 .
$$

Здесь $x$ - пространственная координата, $T$ - температура, $\lambda$ - коэффициент теплопроводности, $Q$ - тепловой эффект химической реакции, $\rho$ - плотность (массовая концентрация) активного компонента, $R$ - газовая постоянная, $k_{0}$ - предэкспоненциальный множитель константы скорости химической реакции, $E$ - энергия активации химической реакции. Энергия активации определяет температурную зависимость скорости реакции, поэтому является основной характеристикой реакционной способности при исследовании условий теплового взрыва.

Используя характерные масштабы задачи, можно переписать это уравнение в следующем виде [2]:

$$
\theta^{\prime \prime}+F k \exp \left(\frac{\theta}{1+\operatorname{Ar} \theta}\right)=0
$$

На границах заданы условия: 


$$
\theta^{\prime}(0)=0, \theta(1)=0 .
$$

Здесь $\theta$ - приведенная температура, Fk - критерий Франк-Каменецкого (отношение скорости реакции при температуре окружающей среды к скорости теплопередачи), $\mathrm{Ar}-$ число Аррениуса (обычно - малый параметр). При критическом значении Fk решение уравнения (2) разрушается, наступает тепловой взрыв.

Нелинейность функции источника не позволяет использовать в качестве функционала производство энтропии или другие связанные с ним интегральные характеристики системы. Можно показать, что минимум производства энтропии будет реализовываться только при $\mathrm{Ar}$ $>>$ 1: в большинстве случаев это условие не выполняется [9]. Однако можно легко показать, что при $\mathrm{Ar} \rightarrow 0$ решение уравнения (1) является экстремалью интеграла:

$$
I=\int_{0}^{1}\left[\frac{1}{2}\left(\theta^{\prime}\right)^{2}-\mathrm{Fk} \exp (\theta)\right] d \xi .
$$

В работе [36] из условия экстремальности интеграла (4) были получены численные оценки критического значения параметра $\mathrm{Fk}$ для системы с двумя параллельными реакциями. Более подробно свойства интеграла (4) были исследованы в недавних работах $[37,38]$ на примере нескольких пробных функций (парабола и косинус), в том числе с учетом зависимости коэффициента теплопроводности от температуры. Вклад первого слагаемого в подынтегральное выражение в (4) положителен, второго слагаемого 0 отрицателен, поэтому существование ограниченного решения (1) определяется существованием локального минимума $I$. При критическом значении $\mathrm{Fk}$ локальный минимум превращается в точку перегиба.

Естественным образом можно распространить задачу минимизации интеграла (4) на систему с произвольным числом экзотермических реакций:

$$
I=\int_{0}^{1}\left[\frac{1}{2}\left(\theta^{\prime}\right)^{2}-\sum_{i} \frac{\mathrm{Fk}_{i}}{s_{i}} \exp \left(s_{i} \theta\right)\right] d \xi .
$$

В пределе можно перейти к непрерывному представлению:

$$
I=\int_{0}^{1}\left[\frac{1}{2}\left(\theta^{\prime}\right)^{2}-\int_{s_{\min }}^{s_{\max }} \frac{\mathrm{Fk}(s)}{s} \exp (s \theta) d s\right] d \xi .
$$

Например, при кинетическом описании динамического поведения некоторых реакционных сред используется приближение непрерывно изменяющейся энергии активации: в сложных многокомпонентных системах (особенно в конденсированной фазе) реакционная способность активных компонентов зависит от взаимного расположения реакционноспособных молекул и их фрагментов, поэтому температурная зависимость скорости реакции становится более сложной [39]. В этом случае лучшим приближением будет использование вместо дифференциального уравнения (2) следующего интегродифференциального уравнения:

$$
\theta^{\prime \prime}+F k \int_{s_{\min }}^{s_{\max }} f(s) \exp \left(\frac{1-s}{\mathrm{Ar}}\right) \exp (s \theta) d s=0
$$

Интегральный функционал, экстремалью которого является решение (7), можно записать так:

$$
I=\int_{0}^{1}\left[\frac{1}{2}\left(\theta^{\prime}\right)^{2}-\mathrm{Fk} \int_{s_{\min }}^{s_{\max }} \frac{f(s)}{s} \exp \left(\frac{1-s}{\mathrm{Ar}}\right) \exp (s \theta) d s\right] d \xi .
$$

Здесь $f(s)$ - это функция распределения, например, гауссова функция от s:

$$
f(s)=\operatorname{Cexp}\left[-\frac{(s-1)^{2}}{2 \sigma^{2}}\right] .
$$

Постоянная $C$ определяется из условия нормировки, а параметр $\sigma$, равный среднему квадратичному отклонению энергии активации от среднего значения, является новым параметром задачи. Отметим, что приближение аррениусовского коэффициента экспоненциальной функцией приведенной температуры в уравнении (7) может давать 
большие погрешности, если $s<<1$. Поэтому естественным условием применимости расчетов на основе уравнения (8) будет достаточно резкое уменьшение $f(s)$ при уменьшении $s$. Физически это условие означает, что реагирующая система должна быть химически устойчивой при низких температурах, т.е. величина $\sigma$ не может быть слишком большой. Естественно, что интеграл (8) переходит в (4) при $\sigma \rightarrow 0$, т.е. для $f(s)=\delta(s-1)$. В общем случае распределение не обязательно должно быть симметричным: в настоящей работе рассматривается лишь один из самых простых частных случаев.

Решение задачи на минимум функционала (8) позволяет исследовать условия возникновения теплового взрыва при наличии флуктуаций, которые изменяют реакционную способность реагирующих молекул. Влияние флуктуаций температуры в неоднородном потоке рассматривалось в работах [40]. Влияние флуктуаций концентрации реагента в дисперсных средах исследовалось в [41]. В настоящей работе рассматривается задача о стационарном распределении температуры, поэтому флуктуации связаны не колебаниями параметров во времени, а с распределением реагирующих частиц по конфигурациям.

3. Результаты расчетов. Для минимизации функционала в уравнении (8) используется пробная функция, удовлетворяющая граничным условиям (3) и содержащая единственный неопределенный параметр:

$$
\varphi(\xi)=a\left(1-\xi^{2}\right) .
$$

Такой выбор функции $\varphi(\xi)$ позволяет получить аналитическое решение при минимизации функционала (4) из условия $d I / d a=0$. Для функционала (8) получить аналитическое решение намного сложнее. Параметр $a$ определяет максимальное значение приведенной температуры, которая обычно имеет порядок единицы при критическом значении Fk. Поскольку исходное уравнение описывает тепловое самовоспламенение, максимум температуры достигается в центре реактора. Параметр Ar равен 0.025, диапазон значений $s$ составляет от 0.01 до 3. Численное интегрирование проводилось методом трапеций: шаг по координате $\xi$ составляет $10^{-3}$, шаг по переменной $s$ тоже $10^{-3}$. Предварительные расчеты показали, что при таком выборе шага численное решение слабо зависит от его величины (расчеты с шагом, меньшим в десять раз, дают незначительное изменение критических параметров).

Критическое значение $\mathrm{Fk}$ (обозначим его $\mathrm{Fk}_{c r}$ ) для задачи (2) равно 0.88. Как указывалось выше, величина интеграла (4) при $\mathrm{Fk}=\mathrm{Fk}_{c r}$ имеет локальный минимум. При величине $\sigma$, близкой к нулю, $\mathrm{Fk}_{c r}$ в задаче (8) остается близким к 0.88. Пробная функция $\varphi$ достаточно хорошо воспроизводит поведение распределение приведенной температуры, но ее использование все же вносит небольшую погрешность при оценке $\mathrm{Fk}_{c r}$. Поэтому $\mathrm{Fk}_{c r}$, определяемое из условия существования точки перегиба на зависимости $I$ от $a$ для уравнения (4), составляет около 0.89. При увеличении дисперсии усиливается вклад реагирования молекул с меньшей энергией активации, поэтому $\mathrm{Fk}_{c r}$ ожидаемо снижается. На рис. 1 приведены расчетные зависимости функционала $I$ в уравнении (8) от $a$ для разных значений $\sigma$ и $\mathrm{Fk}$ вблизи критических значений. На всех графиках виден переход от кривой с локальным минимумом к кривой с монотонно убывающей кривой. Критическое значение $\mathrm{Fk}_{c r}$, как говорилось выше, соответствует кривой с точкой перегиба вблизи $a=1$ (в расчетах значение параметра $a$ варьировалось в пределах от 0 до 3 ).

Зависимость $\mathrm{Fk}_{c r}$ от $\sigma$ представлена на рис. $2 \mathrm{a}:$ при $\sigma=10^{-3}$ функция распределения почти не отличается от $\delta$-функции при выбранной величине шага по $s$, в области $\sigma=10^{-2}-10^{-1}$ происходит резкое снижение, после чего система становится неустойчивой уже при физически нереалистичных значениях $\mathrm{Fk}$. Можно предположить, что с увеличением дисперсии уменьшается эффективная энергия активации реакции. То есть, предполагаем, что 
уравнение (8) можно аппроксимировать уравнением (4), используя функцию распределения $f(s)=\delta(s-\langle s\rangle)$, где $\langle s\rangle=\langle E\rangle / E_{0}-$ эффективное среднее значение коэффициента $s$ при заданном $\sigma$. Из определения параметра $\mathrm{Fk}[1]$ следует, что:

$$
\frac{\mathrm{Fk}}{\mathrm{Fk}_{0}}=\langle s\rangle \exp \left(\frac{\langle s\rangle-1}{\mathrm{Ar}}\right) \text {. }
$$

Небольшое уменьшение энергии активации приводит к значительному ускорению химической реакции: можно приближенно пренебречь изменением $s$ перед экспонентой и рассматривать изменение $s$ только в показателе. Тогда получаем выражение для $\langle s\rangle$ в виде:

$$
\langle s\rangle \simeq 1+\operatorname{Ar} \ln \left(\frac{\mathrm{Fk}}{\mathrm{Fk}_{0}}\right) \text {. }
$$
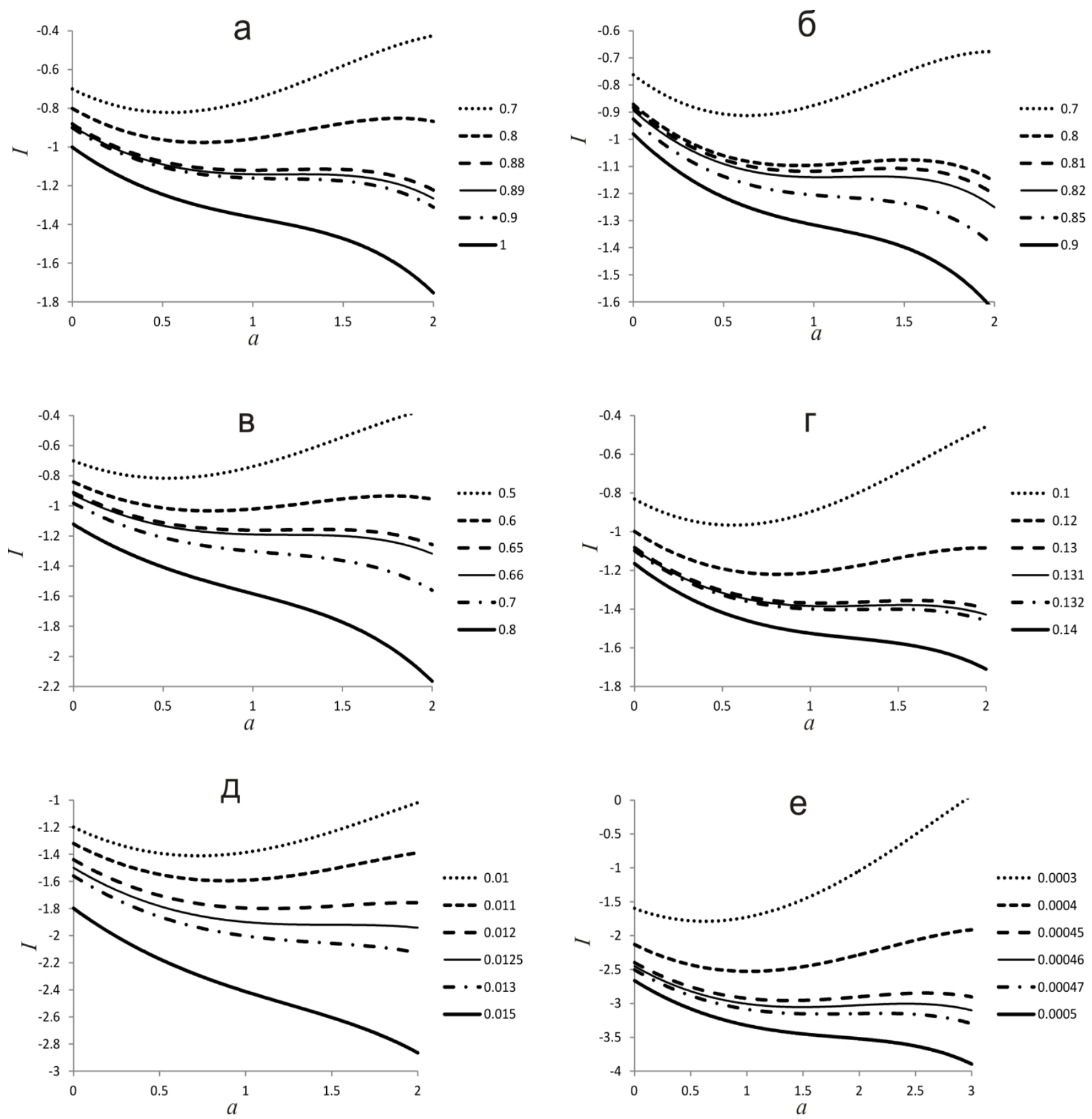

Рис. 1. Расчетные зависимости функционала $I$ от максимальной приведенной температуры $a$ : (а) $\sigma=0.001$; (б) $\sigma=0.01$; (в) $\sigma=0.02$; (г) $\sigma=0.05$; (д) $\sigma=0.075$; (е) $\sigma=0.1$.

Числа на легенде - значения параметра Fk. 
Зависимость $\langle s\rangle$ от $\mathrm{Fk}_{c r}$ (при $\left.\mathrm{Fk}_{0}=0.89\right)$ приведена на рис. $2 \mathrm{~b}$. С увеличением дисперсии до 0.1 происходит снижение эффективной энергии активации примерно на $20 \%$, что и приводит к резкому увеличению скорости реакции (при прочих равных условиях) и, как следствие, быстрой потере тепловой устойчивости. Естественно, что вклад в снижение энергии активации дает лишь область $s<1$, поэтому при рассмотрении несимметричных функций распределения решающее значение будет иметь именно эта часть распределения.

Наконец, отметим, что решение задачи оптимизации интеграла (8) требует намного меньших вычислительных затрат по сравнению с решением исходной краевой задачи, в том числе и благодаря тому, что интеграл (8) существует даже при недопустимых для дифференциального уравнения (7) значениях максимальной температуры. В связи с этим вариационный подход может оказаться полезным и для других, более детальных постановок задачи, связанных с существованием критических условий в системах с химическими реакциями.

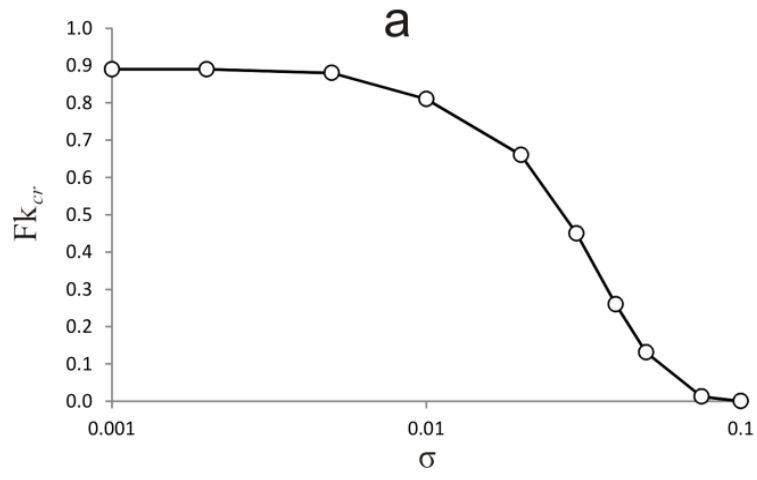

6

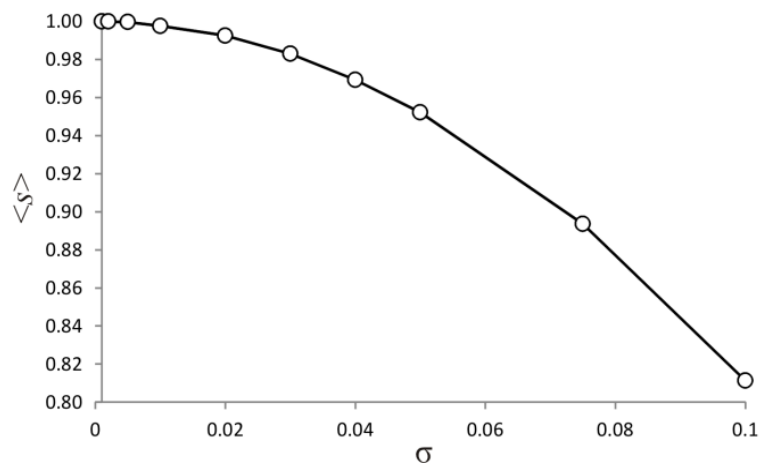

Рис. 2. Зависимость критического значения критерия Франк-Каменецкого и эффективной энергии активации от дисперсии б.

Выводы. В работе предложен численный подход к оценке условий теплового взрыва в системе с флуктуациями реакционной способности на основе решения вариационной задачи. Распределение энергии активации считается гауссовым, а функция источника является средней величиной по этому распределению. Приближенное решение соответствует стационарной точке функционала, зависящего от градиента температуры и среднего значения функции источника. Расчеты показывают, что с увеличением дисперсии распределения происходит снижение эффективной энергии активации химической реакции, поэтому критическое значение критерия Франк-Каменецкого также снижается. Предложен способ оценки эффективной энергии активации по изменению критического значения критерия Франк-Каменецкого.

Благодарности. Работа выполнена в рамках проекта государственного задания FWEU2021-0005 (рег. № АAАА-А21-121012190004-5) программы фундаментальных исследований РФ на 2021-2030 гг. 


\section{СПИСОК ЛИТЕРАТУРЫ}

1. Frank-Kamenetskii D.A. Diffusion and heat exchange in chemical kinetics. Princeton Univ. Press. 2015. 370 p.

2. Kahm W., Vassiliadis V.S. Thermal stability criterion integrated in model predictive control for batch reactors // Chemical Engineering Science. 2018. V. 188. Pp. 192-207. DOI: 10.1016/j.ces.2018.05.032

3. Shechter R.S. The variational method in engineering. McGraw-Hill. 1967. 287 p.

4. Gafiichuk V.V., Lubashevskii I.A. Variational principles of dissipative systems // Journal of Soviet Mathematics. 1993. V. 67. № 2. Pp. 2943-2949. DOI: 10.1007/BF01095874

5. Максимов Г.А. Обобщенный вариационный принцип для диссипативной гидродинамики и механики сплошной среды // Вычислительная механика сплошных сред. 2009. Т. 2. № 4. С. 92-104. DOI: 10.7242/1999-6691/2009.2.4.34

6. Fukugawa H., Fujitani Y. A Variational Principle for Dissipative Fluid Dynamics // Progress in Theoretical Physics. 2012. V. 127. № 5. Pp. 921-935. DOI: 10.1143/PTP.127.921

7. Finlayson B.A., Scriven L.E. On the search for variational principles // International Journal of Heat and Mass Transfer. 1967. V. 10. № 6. Pp. 799-821. DOI: 10.1016/00179310(67)90139-1

8. A variational principle for gradient flows in metric spaces / R. Rossi, G. Savare, A. Segatti, U. Stefanelli // Comptes Rendus Mathematique. 2011. V. 349. № 23-24. Pp. 1225-1228. DOI: 10.1016/j.crma.2011.11.002

9. Glansdorf P., Prigogine I. Thermodynamic theory of structure, stability and fluctuations. NY: Wiley-Interscience. 1971. $305 \mathrm{p}$.

10. Yang C.T. Variational Theories in Hydrodynamics and Hydraulics // Journal of Hydraulic Engineering. 1994. V. 120. № 6. Pp. 737. DOI: 10.1061/(ASCE)0733-9429(1994)120:6(737)

11. Kaganovich B.M., Keiko A.V., Shamansky V.A. Equilibrium thermodynamic modeling of dissipative macroscopic systems // Advances in chemical engineering. Volume 39. Thermodynamics and kinetics of complex systems. Elsevier. 2010. Pp. 1-74. DOI: 10.1016/S0065-2377(10)39001-6

12. Kerner E.H. Dynamical aspects of kinetics // The Bulletin of Mathematical Biophysics. 1964. V. 26. № 4. Pp. 333-349. DOI: 10.1007/BF02484234

13. Орлов В.Н., Розоноэр Л.И. Вариационный принцип для уравнения макроскопической динамики и его приложение в химической кинетике // Журнал вычислительной математики и математической физики. 1981. Т. 21. № 5. С. 1192-1205.

14. Biot M.A. Variational-lagrangian thermodynamics of evolution of collective chemical systems // Chemical Physics. 1978. V. 29. № 1-2. Pp. 97-115. DOI: 10.1016/03010104(78)85064-2

15. Sieniutycz S. Variational thermomechanical processes and chemical reactions in distributed systems // International Journal of Heat and Mass Transfer. 1997. V. 40. № 14. Pp. 34673485. DOI: 10.1016/S0017-9310(96)00235-9

16. Yourgrau W., Raw C.J.G. Variational principles and chemical reactions // Il Nuevo Cimento. 1957. V. 5(3). Pp. 472-478. DOI: 10.1007/BF02743932

17. Sieniutycz S., Shiner J.S. Variational and extremum properties of homogeneous chemical kinetics. I. Lagrangian and Hamiltonian-like formulations // Open Systems \& Information Dynamics. 1992. V. 1. № 2. Pp. 149-182. DOI: 10.1007/BF02228941

18. Быков В.И., Старостин И.Е. Квазиградиентные модели динамики процессов горения в закрытых системах // ДАН. 2013. Т. 451. № 1. С. 53-56. DOI: $10.7868 / \mathrm{S} 0869565213190158$ 
19. Mielke A. A gradient structure for reaction-diffusion systems and for energy-drift-diffusion systems // Nonlinearity. 2011. V. 24. Pp. 1329-1346. DOI: 10.1088/0951-7715/24/4/016

20. Parmon V.N. Catalysis and non-equilibrium thermodynamics: modern in situ studies and new theoretical approaches // Catalysis Today. 1999. V. 51. Pp. 435-456. DOI: 10.1016/S0920-5861(99)00032-2

21. Martyushev L.M., Seleznev V.D. Maximum entropy production: application to crystal growth and chemical kinetics // Current Opinion in Chemical Engineering. 2015. V. 7. Pp. 23-31. DOI: 10.1016/j.coche.2014.10.003

22. Moroz A. A variational framework for nonlinear chemical thermodynamics employing the maximum energy dissipation principle // Journal of Physical Chemistry B. 2009. V. 113. Pp. 8086-8090. DOI: $10.1021 /$ jp9015646

23. Ross J., Corlan A.D., Muller S.C. Proposed Principles of Maximum Local Entropy Production // Journal of Physical Chemistry B. 2012. V. 116. № 27. Pp. 7858-7865. DOI: 10.1021/jp302088y

24. Lebiedz D. Computing minimal entropy production trajectories: An approach to model reduction in chemical kinetics // Journal of Chemical Physics. 2004. V. 120. Pp. 6890. DOI: $10.1063 / 1.1652428$

25. Reinhardt V., Winckler M., Lebiedz D. Approximation of Slow Attracting Manifolds in Chemical Kinetics by Trajectory-Based Optimization Approaches // Journal of Physical Chemistry A. 2008. V. 112. № 8. Pp. 1712-1718. DOI: 10.1021/jp0739925

26. Lebiedz D., Reinhardt V., Siehr J. Minimal curvature trajectories: Riemann geometry concepts for slow manifold computation in chemical kinetics // Journal of Computational Physics. 2010. V. 229. Pp. 6512-6533. DOI: 10.1016/j.jcp.2010.05.008

27. Lebiedz D. Entropy-related extremum principles for model reduction of dissipative dynamical systems // Entropy. 2010. V. 12. Pp. 706-719. DOI: 10.3390/e12040706

28. Pantoleontos G., Basinas P., Skodras G., Grammelis P., J.D. Pinter, Topis S., G.P. Sakellaropoulos A global optimization study on the devolatilisation kinetics of coal, biomass and waste fuels // Fuel Processing Technology. 2009. V. 90. Pp. 762-769. DOI: 10.1016/j.fuproc.2009.03.011

29. Пененко В.В., Цветова Е.А., Пененко А.В. Развитие вариационного подхода для прямых и обратных задач гидротермодинамики и химии атмосферы // Известия РАН. Физика атмосферы и океана. 2015. Т. 51. № 3. С. 358-367. DOI: $10.7868 / \mathrm{S} 0002351515030098$

30. Sirdeshpande A.R., Ierapetritou M.G., Androulakis I.P. Design of flexible reduced kinetic mechanisms // American Institute of Chemical Engineers Journal. 2001. V. 47. № 11. Pp. 2461-2473. DOI: 10.1002/aic.690471110

31. Вольперт B.А., Вольперт Вл.В., Давтян Д.С. Применение минимаксного метода в задачах горения // Физика горения и взрыва. 1990. № 4. С. 3-8.

32. Stevens A., Papanicolaou G., Heinze S. Variational Principles for Propagation Speeds in Inhomogeneous Media // SIAM Journal of Applied Mathematics. 2001. V. 62. № 1. Pp. 129-148. DOI: 10.1137/S0036139999361148

33. Benguria R.D., Depassier M.C. Variational Principles for the Speed of Traveling Fronts of Reaction-Diffusion Equations // Variational and Extremum Principles in Macroscopic Systems. Elsevier. 2005. Pp. 339-354. DOI: 10.1016/B978-008044488-8/50019-9

34. Nicolis G., Prigogine I. Self-organization in nonequilibrium systems. From dissipative structures to order through fluctuations. NY: Wiley-Interscience. 1977. 491 p. 
35. Strieder W., Aris R. Variational Bounds for Problems in Diffusion and Reaction // IMA Journal of Applied Mathematics. 1971. V. 8. № 3. Pp. 328-334. DOI: 10.1093/imamat/8.3.328

36. Graham-Eagle J.G., Wake G.C. Theory of thermal explosions with simultaneous parallel reactions. II. The two- and three-dimensional cases and the variational method // Proceedings of the Royal Society A. Mathematical, Physical and Engineering Sciences. 1985. V. 401. Pp. 195-202. DOI: 10.1098/rspa.1985.0094

37. Зарубин В.С., Кувыркин Г.Н., Савельева И.Ю. Вариационная форма модели теплового взрыва в твердом теле с зависящей от температуры теплопроводностью // Теплофизика высоких температур. 2018. Т. 56. № 2. С. 235-240. DOI: $10.7868 / \mathrm{S} 0040364418020102$

38. Zarubin V.S., Kuvyrkin G.N., Savelyeva I.Y. Variational estimates of the parameters of a thermal explosion of a stationary medium in an arbitrary domain // International Journal of Heat and Mass Transfer. 2019. V. 135. Pp. 614-619. DOI: 10.1016/j.ijheatmasstransfer.2019.02.009

39. Miura K., Maki T. A Simple Method for Estimating $f(E)$ and $k 0(E)$ in the Distributed Activation Energy Model // Energy and Fuels. 1998. V. 12. № 5. Pp. 864-869. DOI: 10.1021/ef970212q

40. Деревич И.В., Галдина Д.Д. Влияние временной структуры флуктуаций температуры газа на воспламенение мелкодисперсных частиц // Теплофизика и аэромеханика. 2016. Т. 23. № 6. С. 935-949.

41. Гринчук П.С. Влияние стохастичности пространственного распределения частиц газовзвеси на распространение фронта пламени // Физика горения и взрыва. 2014. Т. 50. № 3. С. 32-42.

UDK 517.972.5; 544.45

\title{
NUMERICAL ESTIMATION OF CRITICAL CONDITIONS IN THERMAL EXPLOSION PROBLEM FOR A MEDIUM WITH FLUCTUATIONS OF REACTIVITY
}

\author{
Igor G. Donskoy
}

Cand. Sci., Senior Researcher in Laboratory of Thermodynamics, e-mail: donskoy.chem@ mail.ru, Melentiev Energy Systems Institute,

Siberian Branch of the Russian Academy of Sciences

130, Lermontova st., 664033, Irkutsk, Russia.

\begin{abstract}
Annotation. The problem of a thermal explosion in a medium with fluctuations in reactivity, which are specified through the distribution function for the activation energy, is formulated. A variational formulation is proposed for the differential equation describing the stationary temperature distribution in such a medium. Using a simple test function, the dependence of the variational functional on the value of the maximum temperature is numerically investigated. The critical conditions for a thermal explosion correspond to the appearance of an inflection point on this curve. Calculations show that an increase in the dispersion of the reactivity distribution the stability of the system decreases due to shift of the apparent activation energy of the chemical reaction.
\end{abstract}

Keywords. thermal explosion, inverse variational problem, critical conditions, numerical solution

Acknowledgements. The research was carried at the MESI SB RAS under the program for basic research of the SB RAS (project ID FWEU-2021-0005, reg. No. AAAA-A21-121012190004$5)$. 


\section{REFERENCES}

1. Frank-Kamenetskii D.A. Diffusion and heat exchange in chemical kinetics. Princeton Univ. Press. 2015. 370 p.

2. Kahm W., Vassiliadis V.S. Thermal stability criterion integrated in model predictive control for batch reactors // Chemical Engineering Science. 2018. V. 188. Pp. 192-207. DOI: 10.1016/j.ces.2018.05.032

3. Shechter R.S. The variational method in engineering. McGraw-Hill. 1967. 287 p.

4. Gafiichuk V.V., Lubashevskii I.A. Variational principles of dissipative systems // Journal of Soviet Mathematics. 1993. V. 67. № 2. Pp. 2943-2949. DOI: 10.1007/BF01095874

5. Maximov G.A. Obobshchennyy variatsionnyy printsip dlya dissipativnoy gidrodinamiki i mekhaniki sploshnoy sredy [Generalized variational principle in dissipative hydrodynamics and continuum mechanics] // Vychislitel'naya mekhanika sploshnykh sred = Computational Continuum Mechanics. 2009. V. 2. № 4. Pp. 92. DOI: 10.7242/1999-6691/2009.2.4.34

6. Fukugawa H., Fujitani Y. A Variational Principle for Dissipative Fluid Dynamics // Progress in Theoretical Physics. 2012. V. 127. № 5. Pp. 921-935. DOI: 10.1143/PTP.127.921

7. Finlayson B.A., Scriven L.E. On the search for variational principles // International Journal of Heat and Mass Transfer. 1967. V. 10. № 6. Pp. 799-821. DOI: 10.1016/00179310(67)90139-1

8. A variational principle for gradient flows in metric spaces / R. Rossi, G. Savare, A. Segatti, U. Stefanelli // Comptes Rendus Mathematique. 2011. V. 349. № 23-24. Pp. 1225-1228. DOI: $10.1016 /$ j.crma.2011.11.002

9. Glansdorf P., Prigogine I. Thermodynamic theory of structure, stability and fluctuations. NY: Wiley-Interscience. 1971. 305 p.

10. Yang C.T. Variational Theories in Hydrodynamics and Hydraulics // Journal of Hydraulic Engineering. 1994. V. 120. № $6 . \quad$ Pp. $737 . \quad$ DOI: 10.1061/(ASCE)07339429(1994)120:6(737)

11. Kaganovich B.M., Keiko A.V., Shamansky V.A. Equilibrium thermodynamic modeling of dissipative macroscopic systems // Advances in chemical engineering. Volume 39. Thermodynamics and kinetics of complex systems. Elsevier. 2010. Pp. 1-74. DOI: 10.1016/S0065-2377(10)39001-6

12. Kerner E.H. Dynamical aspects of kinetics // The Bulletin of Mathematical Biophysics. 1964. V. 26. № 4. Pp. 333-349. DOI: 10.1007/BF02484234

13. Orlov V.N., Rozonoer L.I. Variatsionnyy printsip dlya uravneniya makroskopicheskoy dinamiki i yego prilozheniye $\mathrm{v}$ khimicheskoy kinetike [Variational principle for macroscopic dynamics equation and it application in chemical kinetics] //Zhurnal vychislitel'noy matematiki $\mathrm{i}$ matematicheskoy fiziki $=$ Journal of Computational Mathematics and Mathematical Physics. 1981. V. 21. № 5. Pp. 1192. [in Russian]

14. Biot M.A. Variational-lagrangian thermodynamics of evolution of collective chemical systems // Chemical Physics. 1978. V. 29. № 1-2. Pp. 97-115. DOI: 10.1016/03010104(78)85064-2

15. Sieniutycz S. Variational thermomechanical processes and chemical reactions in distributed systems // International Journal of Heat and Mass Transfer. 1997. V. 40. № 14. Pp. 34673485. DOI: 10.1016/S0017-9310(96)00235-9

16. Yourgrau W., Raw C.J.G. Variational principles and chemical reactions // Il Nuevo Cimento. 1957. V. 5(3). Pp. 472-478. DOI: 10.1007/BF02743932 
17. Sieniutycz S., Shiner J.S. Variational and extremum properties of homogeneous chemical kinetics. I. Lagrangian- and Hamiltonian-like formulations // Open Systems \& Information Dynamics. 1992. V. 1. № 2. Pp. 149-182. DOI: 10.1007/BF02228941

18. Bykov V.I., Starostin I.E. Kvazigradiyentnyye modeli dinamiki protsessov goreniya v zakrytykh sistemakh [Quasi-gradient models of the dynamics of combustion processes in closed systems] // DAN = Doklady Physical Chemistry. 2013. V. 451. № 1. Pp. 150-153. DOI: $10.1134 /$ S0012501613070026

19. Mielke A. A gradient structure for reaction-diffusion systems and for energy-drift-diffusion systems // Nonlinearity. 2011. V. 24. Pp. 1329-1346. DOI: 10.1088/0951-7715/24/4/016

20. Parmon V.N. Catalysis and non-equilibrium thermodynamics: modern in situ studies and new theoretical approaches // Catalysis Today. 1999. V. 51. Pp. 435-456. DOI: 10.1016/S0920-5861(99)00032-2

21. Martyushev L.M., Seleznev V.D. Maximum entropy production: application to crystal growth and chemical kinetics // Current Opinion in Chemical Engineering. 2015. V. 7. Pp. 23-31. DOI: 10.1016/j.coche.2014.10.003

22. Moroz A. A variational framework for nonlinear chemical thermodynamics employing the maximum energy dissipation principle // Journal of Physical Chemistry B. 2009. V. 113. Pp. 8086-8090. DOI: 10.1021/jp9015646

23. Ross J., Corlan A.D., Muller S.C. Proposed Principles of Maximum Local Entropy Production // Journal of Physical Chemistry B. 2012. V. 116. № 27. Pp. 7858-7865. DOI: 10.1021/jp302088y

24. Lebiedz D. Computing minimal entropy production trajectories: An approach to model reduction in chemical kinetics // Journal of Chemical Physics. 2004. V. 120. Pp. 6890. DOI: $10.1063 / 1.1652428$

25. Reinhardt V., Winckler M., Lebiedz D. Approximation of Slow Attracting Manifolds in Chemical Kinetics by Trajectory-Based Optimization Approaches // Journal of Physical Chemistry A. 2008. V. 112. № 8. Pp. 1712-1718. DOI: 10.1021/jp0739925

26. Lebiedz D., Reinhardt V., Siehr J. Minimal curvature trajectories: Riemann geometry concepts for slow manifold computation in chemical kinetics // Journal of Computational Physics. 2010. V. 229. Pp. 6512-6533. DOI: 10.1016/j.jcp.2010.05.008

27. Lebiedz D. Entropy-related extremum principles for model reduction of dissipative dynamical systems // Entropy. 2010. V. 12. Pp. 706-719. DOI: 10.3390/e12040706

28. Pantoleontos G., Basinas P., Skodras G., Grammelis P., J.D. Pinter, Topis S., G.P. Sakellaropoulos A global optimization study on the devolatilisation kinetics of coal, biomass and waste fuels // Fuel Processing Technology. 2009. V. 90. Pp. 762-769. DOI: 10.1016/j.fuproc.2009.03.011

29. Penenko V.E., Tsvetova E.A., Penenko A.V. Razvitiye variatsionnogo podkhoda dlya pryamykh i obratnykh zadach gidrotermodinamiki i khimii atmosfery [Development of variational approach for direct and inverse problems of atmospheric hydrodynamics and chemistry] // Izvestiya RAN. Fizika atmosfery i okeana = Izvestiya RAN. Atmospheric and Oceanic Physics. 2015. V. 51. Pp. 311. DOI: 10.1134/S0001433815030093

30. Sirdeshpande A.R., Ierapetritou M.G., Androulakis I.P. Design of flexible reduced kinetic mechanisms // American Institute of Chemical Engineers Journal. 2001. V. 47. № 11. Pp. 2461-2473. DOI: 10.1002/aic.690471110

31. Vol'pert V.A., Vol'pert V1.A., Davtyan D.S. Primeneniye minimaksnogo metoda V zadachakh goreniya [Application of a minimax method in combustion problems] // Fizika goreniya i vzryva $=$ Physics of Combustion and Explosion. 1990. V. 26. Pp. 371-376. DOI: 10.1007/BF00745072 
32. Stevens A., Papanicolaou G., Heinze S. Variational Principles for Propagation Speeds in Inhomogeneous Media // SIAM Journal of Applied Mathematics. 2001. V. 62. № 1. Pp. 129-148. DOI: $10.1137 /$ S0036139999361148

33. Benguria R.D., Depassier M.C. Variational Principles for the Speed of Traveling Fronts of Reaction-Diffusion Equations // Variational and Extremum Principles in Macroscopic Systems. Elsevier. 2005. Pp. 339-354. DOI: 10.1016/B978-008044488-8/50019-9

34. Nicolis G., Prigogine I. Self-organization in nonequilibrium systems. From dissipative structures to order through fluctuations. NY: Wiley-Interscience. 1977. 491 p.

35. Strieder W., Aris R. Variational Bounds for Problems in Diffusion and Reaction // IMA Journal of Applied Mathematics. 1971. V. 8. № 3. Pp. 328-334. DOI: 10.1093/imamat/8.3.328

36. Graham-Eagle J.G., Wake G.C. Theory of thermal explosions with simultaneous parallel reactions. II. The two- and three-dimensional cases and the variational method // Proceedings of the Royal Society A. Mathematical, Physical and Engineering Sciences. 1985. V. 401. Pp. 195-202. DOI: 10.1098/rspa.1985.0094

1. 37 Zarubin V.S., Kuvyrkin G.N., Savel'eva I.Y. Variatsionnaya forma modeli teplovogo vzryva $\mathrm{v}$ tverdom tele s zavisyashchey ot temperatury teploprovodnost'yu [The Variational Form of the Mathematical Model of a Thermal Explosion in a Solid Body with Temperature-Dependent Thermal Conductivity] //Teplofizika vysokikh temperature $=$ High Temperature Thermal Physics. 2018. V. 56. Pp. 223-228. DOI: 10.1134/S0018151X18010212

37. Zarubin V.S., Kuvyrkin G.N., Savelyeva I.Y. Variational estimates of the parameters of a thermal explosion of a stationary medium in an arbitrary domain // International Journal of Heat and Mass Transfer. 2019. V. 135. Pp. 614-619. DOI: 10.1016/j.ijheatmasstransfer.2019.02.009

38. Miura K., Maki T. A Simple Method for Estimating $f(E)$ and $k 0(E)$ in the Distributed Activation Energy Model // Energy and Fuels. 1998. V. 12. № 5. Pp. 864-869. DOI: 10.1021/ef970212q

39. Derevich I.V., Galdina D.D. Vliyaniye vremennoy struktury fluktuatsiy temperatury gaza na vosplameneniye melkodispersnykh chastits [Temporal structure of gas temperature fluctuations and ignition of fine particles] // Teplofizika i aeromekhanika $=$ Thermal physics and aeromechanics. 2016. V. 23. Pp. 899-912. DOI: 10.1134/S0869864316060123

40. Grinchuk P.S. Vliyaniye stokhastichnosti prostranstvennogo raspredeleniya chastits gazovzvesi na rasprostraneniye fronta plameni [Effect of stochasticity of the spatial distribution of particles in a gas suspension on combustion front propagation] //Fizika goreniya i vzryva $=$ Physics of Combustion and Explosion. 2014. V. 50. Pp. 272-281. DOI: $10.1134 / \mathrm{S} 0010508214030046$ 